\title{
Patient Safety: What Is Working and Why?
}

Thomas Bartman, $M D, P h D^{1,2, *}$

C. Briana Bertoni, MD, $L S S B B, M B O E^{3}$

Jenna Merandi, PharmD, MS, CPPS

Michael Brady, MD2,5

Ryan S. Bode, $M D^{2,6}$

\author{
Address \\ ${ }^{*}{ }^{*}$ Quality Improvement Services, Nationwide Children's Hospital, 700 Children's \\ Drive, Columbus, $0 \mathrm{H}, 43205$, USA \\ Email: thomas.bartman@nationwidechildrens.org \\ 2Department of Pediatrics, The Ohio State University College of Medicine, $370 \mathrm{~W}$ \\ 9th Ave, Columbus, $\mathrm{OH}, 43210$, USA \\ ${ }^{3}$ Clinical Fellowship in Quality and Safety Leadership, Nationwide Children's Hos- \\ pital, 700 Children's Drive, Columbus, $0 \mathrm{H}, 43205$, USA \\ ${ }^{4}$ Nationwide Children's Hospital, 700 Children's Drive, Columbus, OH, 43205, USA \\ ${ }^{5}$ Patient Safety, Nationwide Children's Hospital, 700 Children's Drive, Columbus, \\ OH, 43205, USA \\ ${ }^{6}$ Hospital Medicine, Nationwide Children's Hospital, 700 Children's Drive, Colum- \\ bus, $\mathrm{OH}, 43205$, USA
}

Published online: 11 April 2019

(C) Springer Nature Switzerland AG 2019

This article is part of the Topical Collection on Patient Safety

Keywords Patient safety · Culture $\cdot$ Event reporting $\cdot$ Electronic health record $\cdot$ Quality improvement $\cdot$ Standardization

Abbreviations IOM Institute of Medicine - ADE Adverse drug event - QI Quality improvement - HAI Healthcare acquired infection - CLABSI Central line-associated bloodstream infection - SSI Surgical site infection - CAUTI Catheter-associated urinary tract infection - VAP Ventilator-associated pneumonia - CDC Centers for Disease Control - HIPAA Health Insurance Portability and Accountability Act - CDS Clinical decision support - ICU Intensive care unit . ISMP Institute for Safe Medication Practices . WAD Work as done - WAI Work as imagined

\begin{abstract}
Purpose of review Our goal is to review a number of methodologies which have been used to improve safety in healthcare since the release of the Institute of Medicine report in 1998 which documented that error was a significant cause of mortality in the USA.

Recent findings Multifaceted approaches have each led to reduction in error. Methods for error reduction included in this review are "Just Culture," increased transparency and accountability, error reporting and investigation, second-victim programs, training in quality and safety methods, standardization and bundles, electronic health records, computerized order entry, barcode scanning, clinical decision support, predictive
\end{abstract}


analytics, and situational awareness. Newer fields with the potential to improve patient safety include human factors engineering, indication-based prescribing, and Safety II. Summary While each intervention has led to incremental improvement, continued expansion of these programs is necessary to eliminate medical error.

\section{Introduction}

As health care continues growing in complexity daily, concerns regarding patient safety grow with it. Over two decades ago the Institute of Medicine (IOM) reported that as many as 98,000 people die annually from preventable harm [1]. A more recent analysis (although controversial and using different methodology than the IOM) suggested that medical errors currently cause 250,000 American deaths each year which would make error the third leading cause of death second to cancer and heart disease [2]. Regardless of the true incidence of medical error leading to harm, only one goal is clearly acceptable to any individual patient or family-zero preventable harm $[3,4]$. We must achieve this in the context of patients living longer with multiple comorbidities and acute conditions and having higher expectations for quality of care. Increased awareness of our deficiencies in patient safety has led to numerous strategies for improving the quality and safety of care despite trends towards increased disease severity. This review covers many of these strategies; some are generalizable while others are more specific. Finally, we suggest a few up-and-coming areas which will potentially drive safety results further.

\section{"Just culture"}

Perhaps the most important element for improving patient safety is organizational culture. Hospitals with a "Just Culture" share accountability in doing the right thing, both in leadership and the frontline staff. The "Just Culture" focuses error-prevention efforts on improving system design and behavioral choices [5]. A "Just Culture" recognizes that humans are destined to make mistakes [6], and drift at times into at-risk behaviors, no matter how well hospitals design their systems. However, "Just Culture" requires leaders and management to pursue follow-up actions that correct errant behaviors irrespective of those involved or the outcome of a particular error event. When management successfully differentiates between human error, at-risk behavior, and reckless behavior, appropriate actions are taken which may include consoling, coaching, or disciplining the individual involved [7].

\section{Transparency/accountability}

Organizations which desire to create a high-safety culture must emphasize transparency-open disclosure of information regarding safety and safety events with patients, the public, and other healthcare providers [8]. Without transparency, healthcare providers have no opportunity to learn from each other's mistakes, which increases the odds of similar errors in the future [8]. Some organizations have recognized the need for transparency and publish 
their quality and safety results on the Internet, which may drive patient choice and influence staff buy-in to improve safety outcomes [9]. Becoming a "highreliability organization" (one that operates in a complex, highly-hazardous domain for an extended period without failure) requires that hospitals dedicate themselves to consistent safety accountability and effective patient-centered care [10].

\section{Event reporting and investigation}

Frontline employees must identify and report events so problematic issues can be addressed [1]. As a hospital's safety culture matures, an increase in voluntary reporting may occur along with other ways of detecting adverse events such as trigger tool analysis [11]. Increased event identification through reporting and triggers, followed by robust analysis such as rootcause analysis identifies individual and system failures, and improves the ability to understand not only the gaps in our systems, which led to an event, but also to identify and rectify latent errors-errors in waiting [12]. After a harmful event, methods such as the "huddle" process encourage the gathering of interdisciplinary teams who examine what specifically happened and determine appropriate remedial actions to prevent reoccurrence [13]. Hospitals have been successful in utilizing the huddle to identify interventions at the individual, unit, or system level to reduce adverse drug events (ADEs) $[14,15]$. Event reporting and investigation is best applied as a multifaceted approach. One institution, which reduced harmful ADEs by approximately $80 \%$, suggested four critical factors for success-a robust internal quality collaborative model, focus on medication management failure modes, ADE huddles, and increased event reporting in an evolving safety culture [16]. Institutions which hope to achieve higher levels of voluntary event reporting must recognize that the "Just Culture" described previously is critical to making employees feel safe to report instead of cover-up problems.

\section{Second victim programs}

Despite efforts to reduce and eliminate preventable harm, errors-sometimes tragic-will still occur. Healthcare professionals are often abandoned while dealing with the emotional impact following these unfortunate incidents and can have trouble coping with their emotions and reactions $[17,18]$. Wu coined the term "second victim," defined as "those who suffer emotionally when the care they provide leads to patient harm [19]." Years later, the definition was expanded by Scott and colleagues to include those "healthcare providers involved in unanticipated adverse patient events, medical error, and/or patient-related injury who become traumatized by the event [20]." "Second victims" go through distinct recovery stages following these events [21], and institutionally designed programs providing peer support can reduce emotional distress and improve professional quality-of-life [21, 22]. Second victims often desire support from a coworker, peer, or an individual who helps them learn from their mistake and decreases the involved worker from feeling 
isolated $[21,23,24]$. Hospitals must find ways to rapidly respond to grieving healthcare providers and make accessible support mechanisms swiftly available [21,25]. Healthcare providers often suffer in silence, and if we desire to continue improving patient safety, we must improve employee safety as well.

\section{Training in quality and safety methods}

\section{Standardization/bundles}

Institutions can accelerate patient safety improvement efforts through motivated interdisciplinary teams, which include physicians, nurses, pharmacists, administrators, risk managers, and other individuals in place, to help organizations make improvements going forward [26]. Though many of these individuals might have the knowledge and skill to lead change [26], some believe standardized quality improvement (QI) training in areas such as change management and teamwork is necessary for institution-wide QI [27]. One study evaluated four core areas: QI knowledge, testing and implementing change using teams, data management and analysis, and spreading and sustaining science following a QI essentials course [28]. Teaching the Institute for Healthcare Improvement Model for Improvement [29], the authors found that having interdisciplinary teams participating in the course made drastic improvements in QI competency over time and increased QI productivity [28]. Training hundreds of mid-level and senior leaders in a project-based course has created a "can do" QI culture in the institution. Continued execution of QI training programs will enable more individuals throughout health-systems to practice improvement methods towards increasing care quality and reducing patient harm. The expected result is organizations will build a solid "bottomup" foundation for QI while supporting high-reliability at the system level [30].

Care standardization, often through bundled implementation, speaks to a fundamental definition of quality as a reduction in variation [31]. Standardization has achieved reductions in a critical function that has harmed patients since the origin of healthcare-Healthcare-Associated Infections (HAIs). Initially, HAIs resulted from poor hygiene and sanitation and failure to understand germ theory. More recently, HAIs have been associated with surgical procedures, use of invasive devices, and therapies adversely affecting the immune system. When the device-related HAIs began to occur, physicians rationalized these infections as the cost of caring for critically ill patients. Central line-associated bloodstream infections (CLABSI), surgical site infections (SSI), catheterassociated urinary tract infections (CAUTI), and ventilator-associated pneumonia (VAP) are the most common and most serious HAIs. Since not all patients with invasive devices develop HAIs, specific patient characteristics and device care-related activities identified risk factors enhancing or reducing an individual patient's likelihood of acquiring an HAI. Understanding these risk factors led to interventions that have significantly reduced HAIs.

For each HAI, specific interventions were trialed, usually "bundled" together, reducing contamination of a previously sterile or clean site during surgery or device insertion and minimizing indwelling device manipulation to reduce 
subsequent contamination. The resulting insertion and maintenance "bundles" for each device-associated HAI are evidence-based and standardized [32-37]. Because the risk for a device-related HAI increases while any invasive device remains in a patient, all device maintenance bundles require a daily conversation to determine if the device is still essential. This practice ensures removal as soon as feasible when devices are no longer medically necessary.

Insertion and maintenance bundles vary by device. For each device, the use of standardized bundles for insertion and maintenance requires high compliance levels for full effectiveness. The Centers for Disease Control and Prevention (CDC) through its National Healthcare Safety Network determined that intensive care units (ICUs) utilizing these standardized insertion and maintenance bundles reduce CLABSIs by at least $38 \%$ (see Table 1 for one bundle example). However, achieving this magnitude of CLABSI rate required compliance at $>$ $95 \%$ for all bundle elements [38].

In 2009, the eight children's hospitals in Ohio created Solutions for Patient Safety [39] as a network to improve patient safety by utilizing evidence-based practices, such as the HAI bundles, to reduce patient harm. The network has gone national, and even international, with more than 130 children's hospitals participating. Since 2011, with standardized bundle use, the network hospitals have reduced SSIs by $31.2 \%$, CAUTIs by $58.8 \%$, and CLABSIs by $12.1 \%$ [40 $]$.

Evaluations of the impact of each specific device-related bundles were conducted as multi-modal interventions and not an assessment of individual specific bundle elements. Therefore, the benefit of each element is not established. Since the bundle elements add cost and provider time, identifying which bundle elements provide benefit and which do not would be valuable. However, bundle success may preclude efforts to assess individual bundle elements due to concerns that eliminating any bundle element could result in potential patient harm.

\section{Electronic health records (EHRs)}

In the past 19 years, health information technology (HIT) advances have significantly contributed to patient safety. Perhaps the most widely adapted advancements are the EHR and computerized physician order entry (CPOE). Though EHRs first appeared in the 1960s [41], widespread adoption took

Table 1. CLABSI prevention bundle elements

\begin{tabular}{ll}
\hline Insertion & Maintenance \\
Hand hygiene & Daily discussion of line necessity and functionality \\
Chlorhexidine scrub & Regular assessment of dressing integrity \\
No iodine treatment & Standardized access procedure \\
Prepackaged or filled cart, tray, box & Standardized dressing, cap and tubing change (procedures and timing) \\
Insertion checklist & Daily chlorhexidine bathing \\
Full sterile barrier for providers and patients & \\
Insertion training for providers &
\end{tabular}




\section{Computerized order entry (CPOE)}

significantly longer due to some concerns, mostly related to cost, feasibility, and security. In 2009, 10 years after the "To Err is Human" publication by the IOM, the federal government passed the Health Information Technology for Economic and Clinical Health (HITECH) Act [42], which afforded incentives for HIT use, including EHR use. The HITECH Act also expanded the Health Insurance Portability and Accountability Act (HIPAA) to provide coverage for electronic-protected health information. This act catalyzed EHR adoption, which doubled from 34.8\% of hospitals in 2007 to more than $71 \%$ in 2012 [43].

With HIT development, concerns about design problems, workflow disruption, and general dissatisfaction are numerous [44]. Thus, the use of a humanfactors approach (see below) to the design and development of further technology encourages careful evaluation and risk mitigation before EHR implementation is necessary. Despite safety concerns arising from EHR implementation, its application has been linked to enhanced vaccination rates [45] and improved health care maintenance [46] in pediatrics and decreased inpatient mortality with enhanced use of auto-populated notes and records [47].

CPOE development, an advancement of EHR technology, has increased efficiency and safety, particularly when coupled with clinical decision support (CDS) tools. With nearly five billion drug treatment orders written annually, CPOE provides a "clear" alternative to hand-written orders [48]. CPOE targets medication-prescribing errors, which are believed to be the most prevalent ADEs in hospitals $[48,49]$. Utilizing CPOE can detect medication errors more efficiently and automatically by checking that the medication is prescribed at the appropriate dose; further, electronic order delivery increases pharmacy efficiency.

Likewise, adding CDS to CPOE improves adherence to the medication formulary and reduces ADEs by checking prescribed medications against a patient's listed allergies or adverse reactions as well as automatically checking for drug-drug interactions [48]. CDS may also use warnings or hard stops, which limit medication doses to an appropriate range based on a patient's age or size or may require high-risk medications (e.g., anticoagulants or insulin) be ordered with appropriate monitoring.

Lastly, in the outpatient setting, CPOE can increase medication pick-up, particularly when linked to an automatic notification to primary care providers regarding non-compliance [48]. CPOE employment has been associated with reduced hospital mortality for pediatric patients [50].

\section{Barcode scanning}

A further advancement, barcode scanning, has addressed medication administration errors, which were unchanged with CPOE [49]. Before medication administration, a nurse scans both the medication and patient identification band barcodes, which link to an order in the EHR. The administrator is warned not to proceed if the patient does not have an order for the specific medication, including dose and route, due at that time. Using barcode scanning has reduced 
wrong medication, wrong patient, wrong time, wrong route, and wrong-dose errors. Though the five rights of medication administration will never be replaced, barcode scanning, when used appropriately, can act as a defensive wingman to those administering medications. While not all medication errors lead to patient harm, and to our knowledge, no study has shown reduction in hospital-wide preventable harm or serious safety events after introduction of barcode scanning, at least one unit has shown a reduction in preventable adverse drug events upon introduction of this technology [51].

Advances in HIT are just beginning to address pump programming errors. While smart pumps using dosing error reduction software can decrease some programming errors, many medication-infusion administration errors are still possible, including wrong rate, wrong concentration, wrong medication, and wrong dose. Smart pump integration links smart pumps to the EHR, such that medication administration requires barcode scanning the patient's wristband, the medication, and the pump channel. The pump is then automatically programmed using the available order from the linked EHR. Also, the pump automatically administers the medication volume documented in the EHR at the appropriate time, allowing for improved documentation. While relatively new, smart pumps have the opportunity to decrease ADEs by decreasing the keystrokes by approximately $86 \%$ [52] and improving pharmacy workflow by automatically notifying the pharmacy when a bag or syringe is at a pre-specified level and needs to be replaced. As of January 2018, approximately $99 \%$ of hospitals reported using smart pumps for IV medication administration, yet only $15 \%$ used the pump's interoperability function [53]. We anticipate seeing greater evidence of improvement in patient outcomes as smart pump programming links become increasingly adopted.

\section{Clinical decision support (CDS)/predictive analytics}

The most recent introduction to the HIT arena includes the development of predictive analytics. Enhanced EHR use coupled with other IT advances, including wearable devices, created massive amounts of healthcare data. Predictive analytics, a branch of healthcare analytics, uses empirical methods to develop predictions [54]. Despite being in the infancy of its development in healthcare, predictive analytics has been used to forecast cardiac arrest in pediatric intensive care unit (PICU) patients [55•], nephrotoxic-associate acute kidney injury [56], and cardiovascular deterioration in children with hypoplastic left-heart and similar cardiac lesions [57]. CDS and predictive analytics have improved the early detection of sepsis in the ED with an automated solution requiring no additional documentation, identifying patients $68 \mathrm{~min}$ earlier when compared to the previous paper process [58]. A predictive model has been developed to detect pediatric inpatient deterioration outside of the ICU setting up to $24 \mathrm{~h}$ before the event occurs (event being code blue, emergent PICU transfer, or patient death) with $85 \%$ sensitivity and less than a $2 \%$ false positive rate (Hoffman, J. - personal communication). Overall, predictive analytics' ability to act as the "wingman" for busy and distracted healthcare providers may lead to transformational improvement in patient safety. One area which should receive continued exploration is how behavioral economics - the effects of psychological, cognitive, emotional, cultural, and 
social factors on the economic decisions of individuals and institutions and how those decisions vary from those implied by classical theory-can be adapted to non-economic decisions in healthcare and how these factors affect CDS [59].

\section{Situational awareness}

Hospitalized pediatric patients with unrecognized clinical deterioration and failed care escalation resulting in a code blue outside the ICU have an associated increase in mortality. Initial studies demonstrated a $40-67 \%$ mortality with a 1 -year survival rate of $15 \%$ [60]. With more timely and effective resuscitation via pediatric code teams, both event survival and survival to discharge have improved significantly without a corresponding increase in long-term neurological deficits [61]. Rapid response team (RRT) development has effectively led to rescuing clinical deterioration and resulted in decreased codes outside the ICU and overall hospital mortality. Initially demonstrated in a single pediatric facility, RRTs resulted in decreased in codes outside the ICU by $72 \%$ and a decrease in hospital mortality by $18 \%$ [62]; two systematic reviews have recently affirmed these findings $[63,64]$. However, RRT interventions usually represent a delayed recognition of ongoing patient deterioration and subsequent rescue. Hospital systems are challenged to effectively intervene sooner towards a more proximal capture of patient deterioration. This requires altering the trajectory of the decompensating hospitalized pediatric patient with prediction, identification, mitigation, and escalation of at-risk patients.

Recent improvement efforts have focused on increasing situational awareness [65], a term borrowed from the military and aviation industry, and which can be defined and summarized with three questions: (1) What is going on (with the patient/family, the unit, and providers)? (2) What does it mean (data points, analysis, and interpretation)? and (3) What is the predicted outcome (and potential mitigation and escalation plans)?

Code blue teams and RRTs are system solutions targeting increasing situational awareness, albeit interventions at the end of the deterioration spectrum. Awareness and prediction tools such as the pediatric early warning scores (PEWS) have been well studied including most recently and robustly in a large multicenter prospective randomized control study, which did not show that PEWS significantly affected hospital mortality [66]. However, PEWS initiation did result in a decrease in defined "significant clinical deterioration events," which represented a more proximal capture of decompensation on the floor. Similarly defined events, also referred to as unsafe or emergency transfers, are eightfold more common than code blue events and associated with increased morbidity and mortality compared to other ICU transfer patients [67]. Recent research and technological innovations have focused on electronic health record predictive analytic tools with several models outperforming PEWS in recognizing clinical deterioration for hospitalized patients [68]. In conjunction with more accurate predictive capacity, innovative models emphasizing situational awareness with earlier identification, mitigation, and escalation have favorably impacted unrecognized clinical deterioration [69, 70]. 
Preventing delayed or unrecognized clinical deterioration is a complex problem requiring complex solutions. A multifaceted approach including organizational, operational, cultural, technical, and human factor solutions are necessary to realize and sustain improvement.

\section{Future directions—-human factors engineering (HFE)}

HFE is the "scientific discipline concerned with understanding interactions among humans and other elements of a system [71]." As such, HFE recognizes that the systems in which we work require complex interplay between humans (care providers) and equipment, supplies, and physical spaces. Efforts to optimize the latter elements without considering the role of fallible humans will limit gains seen through safety improvement efforts. HFE can be divided into the fields of physical ergonomics (how humans move through their workspace), cognitive ergonomics (how human interaction with technology affects decisionmaking), and macro ergonomics (how culture and organizational factors affect human performance). Since 2005, the IOM has been calling for increased HFE consideration to improve patient safety [72]. A popular model for approaching HFE work in healthcare is the Systems Engineering Initiative for Patient Safety (SEIPS) model [73] for which an update has recently been introduced [SEIPS 2.0, [74]]. In SEIPS 2.0, the humans at the center of the work system include both providers and patients/families, the tools/technology, tasks, internal and external environments with which they interact, and the wider organization. The model suggests that the configuration of the components creates specific interactions for any particular process and that process performance depends on the interactions more than individual components. One HFE example is having each physical item required for each step of a task arrayed in sequence from left to right in an equipment tray, thus minimizing or preventing inadvertent skipping of a task. Another HFE example is a "forcing function" which prevents performing one step until a prerequisite step is completed; for example, the OR case start time cannot be entered into the EHR until a box has been checked indicating completion of a procedural time-out.

\section{Future directions_-indication-based prescribing}

Most practitioners are already familiar with the " 5 Rights" of medication safety-right patient, drug, dose, time, and route. In the last few years, especially with the advent of $\mathrm{CPOE}$, a sixth right has been proposed - the right indication [75]. While some initial studies indicated that including the indication for the medication in the electronic order is effective at reducing medical errors [7678], widespread adoption of this technology is lagging. An editorial from the Institute for Safe Medication Practices (ISMP) [79] suggests indication-based prescribing can provide multiple benefits. These advantages include, but are not limited to, preventing errors by narrowing medication choices, especially with look-alike drug names; empowering patients by helping them understand which medication they are taking for which purpose; improving 


\section{Future directions-Safety II}

The predominant method for improving safety in healthcare over the last decades has been learning from our mistakes and redesigning systems to prevent recurrence. In this model, called Safety I, learning opportunities, which drive improvement, become limited as adverse events decrease. Furthermore, as the more common failure modes are eliminated, future events tend to be unrelated to past events and thus cannot be prevented due to past learning. If our goal is zero harm (perfect safety), a shift in our thinking must occur.

Safety II focuses on learning from what goes right and allowing individuals and systems to become more resilient. Hollnagel, Wears, and Braithwaite (2015) in their seminal whitepaper called for a shift towards Safety II [80•]. One key concept from Safety II is understanding how the actual work on the front-lines differs from what is imagined by those not on the front-lines [work as done (WAD) vs. work as imagined (WAI)] [81]. The reason for the differences can be common and mundane-such as a nurse simply triaging and resequencing tasks to get through a busy shift [82], or responding to the introduction of new technology in her workflow [83]. Alternatively, differences between WAD and WAI can be responses to major events where front-line workers recognize a need to change their practices to avoid error. In these cases, a resilient response likely involves a four-step process of monitoring a situation, anticipating impending problems, responding effectively, and learning from successes achieved [84]. While Safety II is still an emerging field, and no reports of Safety II being created de novo in a healthcare setting are available, a recent paper described how Safety II seems to occur in a high-functioning unit and may lead towards strategies for expanding a Safety II framework in other microsystems [85].

\section{Conclusion}

The many strategies described here have achieved incremental improvement in patient safety, which were often proven effective on a small scale (in individual hospitals, or microsystems within those hospitals). Unfortunately, the evidence does not support dramatic improvements in patient safety across our American healthcare system. The disconnect in the data may be due to the limited effect of the current strategies, narrow adoption of these strategies, or decreased safety due to other causes which are negating the current gains. Hopefully, increased spread of effective practices and the addition of more profoundly effective novel strategies will soon lead to marked improvement in patient outcomes. 


\section{Compliance with Ethical Standards}

\section{Conflict of Interest}

Thomas Bartman, declares that he has no conflict of interest. C. Briana Bertoni declares that she has no conflict of interest. Jenna Merandi declares that she has no conflict of interest. Michael Brady declares that he has no conflict of interest. Ryan S. Bode declares that he has no conflict of interest.

Human and Animal Rights and Informed Consent

This article does not contain any studies with human or animal subjects performed by any of the authors.

\section{References}

Papers of particular interest, published recently, have been highlighted as:

- Of importance

1. Kohn LT, Corrigan J, Donaldson MS, Institute of Medicine (U.S.). Committee on Quality of Health Care in America. To err is human: building a safer health system. Washington, D.C.: National Academy Press; 2000.

2. Makary MA, Daniel M. Medical error-the third leading cause of death in the US. BMJ. 2016;353:i2139.

3. Brilli RJ, McClead RE, Davis T, Stoverock L, Rayburn A, Berry JC. The preventable harm index: an effective motivator to facilitate the drive to zero. J Pediatr. 2010;157(4):681-3. https://doi.org/10.1016/j.jpeds. 2010.05.046.

4. Crandall WV, Davis JT, McClead R, Brilli RJ. Is preventable harm the right patient safety metric? Pediatr Clin N Am. 2012;59(6):1279-92. https://doi.org/10. 1016/j.pcl.2012.09.003.

5. Marx D. Patient safety and the "just culture": a primer for health care executives. New York: NY; 2001.

6. Boysen PG. Just culture: a foundation for balanced accountability and patient safety. Ochsner J. 2013;13(3):400-6.

7. Leonard MW, Frankel A. The path to safe and reliable healthcare. Patient Educ Couns. 2010;80(3):288-92. https://doi.org/10.1016/j.pec.2010.07.001.

8. Leape L, Berwick D, Clancy C, Conway J, Gluck P, Guest J, et al. Transforming healthcare: a safety imperative. Qual Saf Health Care. 2009;18(6):424-8. https://doi.org/10.1136/qshc.2009.036954.

9. Bartman T, Carroll B, Alessandrini E, Payne NR. Transparency in pediatric outcomes reporting-reducing knowledge asymmetries in pediatric healthcare. Curr Treat Options in Pediatr. 2015;1(4):320-33. https:// doi.org/10.1007/s40746-015-0029-1.

10. Weick KE, Sutcliffe KM, Obstfeld D. Organizing for high reliability: processes of collective mindfulness. Res Organ Behav. 1999;21:81-123.

11. Rozich JD, Haraden CR, Resar RK. Adverse drug event trigger tool: a practical methodology for measuring medication related harm. Qual Saf Health Care. 2003;12(3):194-200.
12. Root Cause Analysis. Agency for Healthcare Research and Quality. 2018. https://psnet.ahrq.gov/primers/ primer/10. Accessed October 18, 20182018.

13. Wilbur K, Scarborough K. Medication safety huddles: teaming up to improve patient safety. Can J Hospital Pharm. 2005;58(3):151-5https://www.cjhp-online. ca/index.php/cjhp/article/view/307. Accessed on 15 Jan 2019.

14. Merandi J, Morvay S, Lewe D, Stewart B, Catt C, Chanthasene PP, et al. Improvement of medication event interventions through use of an electronic database. Am J Health Syst Pharm. 2013;70(19):1708-14. https://doi.org/10.2146/ajhp130021.

15. Morvay S, Lewe D, Stewart B, Catt C, McClead RE, Brilli RJ. Medication event huddles: a tool for reducing adverse drug events. Jt Comm J Qual Patient Saf. 2014;40(1):39-45.

16. McClead RE, Catt C, Davis JT, Morvay S, Merandi J, Lewe $\mathrm{D}$, et al. An internal quality improvement collaborative significantly reduces hospital-wide medication error related adverse drug events. J Pediatr. 2014;165(6):1222-9.e1. https://doi.org/10.1016/j. jpeds.2014.08.063.

17. Krzan KD, Merandi J, Morvay S, Mirtallo J. Implementation of a "second victim" program in a pediatric hospital. Am J Health Syst Pharm. 2015;72(7):563-7. https://doi.org/10.2146/ajhp140650.

18. Edrees HH, Paine LA, Feroli ER, Wu AW. Health care workers as second victims of medical errors. Pol Arch Med Wewn. 2011;121(4):101-8.

19. Wu AW. Medical error: the second victim. The doctor who makes the mistake needs help too. BMJ. 2000;320(7237):726-7.

20. Scott SD, Hirschinger LE, Cox KR, McCoig M, Brandt J, Hall LW. The natural history of recovery for the healthcare provider "second victim" after adverse patient events. Qual Saf Health Care. 2009;18(5):32530. https://doi.org/10.1136/qshc.2009.032870. 
21. Scott SD, Hirschinger LE, Cox KR, McCoig M, HahnCover K, Epperly KM, et al. Caring for our own: deploying a systemwide second victim rapid response team. Jt Comm J Qual Patient Saf. 2010;36(5):233-40.

22. Winning AM, Merandi JM, Lewe D, Stepney LMC, Liao NN, Fortney CA, et al. The emotional impact of errors or adverse events on healthcare providers in the NICU: the protective role of coworker support. J Adv Nurs. 2018;74(1):172-80. https://doi.org/10.1111/jan.13403.

23. Christensen JF, Levinson W, Dunn PM. The heart of darkness: the impact of perceived mistakes on physicians. J Gen Intern Med. 1992;7(4):424-31.

24. Engel KG, Rosenthal M, Sutcliffe KM. Residents' responses to medical error: coping, learning, and change. Acad Med. 2006;81(1):86-93.

25. Merandi J, Liao N, Lewe D, Morvay S, Stewart B, Catt C, et al. Deployment of a second victim peer support program: a replication study. Pediatr Qual Saf. 2017;2(4):e031. https://doi.org/10.1097/pq9. 0000000000000031.

26. Leape LL, Berwick DM. Five years after to err is human: what have we learned? JAMA. 2005;293(19):2384-90. https://doi.org/10.1001/jama.293.19.2384.

27. Kaplan HC, Provost LP, Froehle CM, Margolis PA. The model for understanding success in quality (MUSIQ): building a theory of context in healthcare quality improvement. BMJ Qual Saf. 2012;21(1):13-20. https:// doi.org/10.1136/bmjqs-2011-000010.

28. Bartman T, Heiser K, Bethune A, Crandall W, McClead $\mathrm{R}, \mathrm{Davis} \mathrm{JT}$, et al. Interprofessional QI training enhances competency and QI productivity among graduates: findings from Nationwide Children's Hospital. Acad Med. 2018;93(2):292-8. https://doi.org/10. 1097/ACM.0000000000001862.

29. Langley GJ. The improvement guide : a practical approach to enhancing organizational performance. 2nd ed. San Francisco: Jossey-Bass; 2009.

30. Scoville R, Little K, Rakover J, Luther K, Mate K. Institute for Healthcare Improvement: Sustaining Improvement. 2016.

31. Montgomery DC. Introduction to statistical quality control. 7th ed. Hoboken, NJ: Wiley; 2013.

32. Berríos-Torres SI, Umscheid CA, Bratzler DW, Leas B, Stone EC, Kelz RR, et al. Centers for Disease Control and Prevention guideline for the prevention of surgical site infection, 2017. JAMA Surg. 2017;152(8):784-91. https://doi.org/10.1001/jamasurg.2017.0904.

33. Boyce JM, Pittet D, Committee HICPA, Force

HSAIHHT. Guideline for hand hygiene in health-care settings. Recommendations of the Healthcare Infection Control Practices Advisory Committee and the HICPAC/SHEA/APIC/IDSA Hand Hygiene Task Force. Society for Healthcare Epidemiology of America/ Association for Professionals in Infection Control/ Infectious Diseases Society of America. MMWR Recomm Rep. 2002;51(RR-16):1-45.

34. Gould CV, Umscheid CA, Agarwal RK, Kuntz G, Pegues DA, Committee HICPA. Guideline for prevention of catheter-associated urinary tract infections 2009. Infect
Control Hosp Epidemiol. 2010;31(4):319-26. https:// doi.org/10.1086/651091.

35. Mangram AJ, Horan TC, Pearson ML, Silver LC, Jarvis WR. Guideline for prevention of surgical site infection, 1999. Hospital Infection Control Practices Advisory Committee. Infect Control Hosp Epidemiol. 1999;20(4):250-78. https://doi.org/10.1086/501620.

36. O'Grady NP, Alexander M, Burns LA, Dellinger EP, Garland J, Heard SO, et al. Guidelines for the prevention of intravascular catheter-related infections. Am J Infect Control. 2011;39(4 Suppl 1):S1-34. https://doi. org/10.1016/j.ajic.2011.01.003.

37. Tablan OC, Anderson LJ, Besser R, Bridges C, Hajjeh R, $\mathrm{CDC}$, et al. Guidelines for preventing health-careassociated pneumonia, 2003: recommendations of CDC and the Healthcare Infection Control Practices Advisory Committee. MMWR Recomm Rep. 2004;53(RR-3):1-36.

38. Furuya EY, Dick A, Perencevich EN, Pogorzelska M, Goldmann D, Stone PW. Central line bundle implementation in US intensive care units and impact on bloodstream infections. PLoS One. 2011;6(1):e15452. https://doi.org/10.1371/journal.pone.0015452.

39. Lyren A, Brilli R, Bird M, Lashutka N, Muething S. Ohio Children's Hospitals' solutions for patient safety: a framework for pediatric patient safety improvement. J Healthc Qual. 2016;38(4):213-22. https://doi.org/10. 1111/jhq.12058.

40. Lyren A, Brilli RJ, Zieker K, Marino M, Muething S, Sharek PJ. Children's hospitals'dolutions for patient safety vollaborative impact on hospital-acquired harm. Pediatrics. 2017;140(3):20163494. https://doi.org/10. 1542/peds.2016-3494

This paper demonstrates that a collaborative of 33 children's hospitals was able to reduce serious safety events by $50 \%$ through the use of local improvement teams.

41. A history of EHRs: 10 things to know. Becker's Health IT \& CIO Report. https://www.beckershospitalreview.com/ healthcare-information-technology/a-history-of-ehrs10-things-to-know.html. Accessed October 18, 2018.

42. Burde H. Health law the hitech act-an overview. Virtual Mentor. 2011;13(3):172-5. https://doi.org/10.1001/ virtualmentor.2011.13.3.hlaw1-1103.

43. National Center for Health Statistics Fact Sheet, 2018. https://www.cdc.gov/nchs/data/factsheets/factsheet_ nhcs.pdf. Accessed October 18, 2018.

44. Karsh BT. Beyond usability: designing effective technology implementation systems to promote patient safety. Qual Saf Health Care. 2004;13(5):388-94. https://doi.org/10.1136/qhc.13.5.388.

45. Fiks AG, Grundmeier RW, Biggs LM, Localio AR, Alessandrini EA. Impact of clinical alerts within an electronic health record on routine childhood immunization in an urban pediatric population. Pediatrics. 2007;120(4):707-14. https://doi.org/10.1542/peds. 2007-0257.

46. Adams WG, Mann AM, Bauchner H. Use of an electronic medical record improves the quality of 
urban pediatric primary care. Pediatrics.

2003;111(3):626-32.

47. Amarasingham R, Plantinga L, Diener-West M, Gaskin DJ, Powe NR. Clinical information technologies and inpatient outcomes: a multiple hospital study. Arch Intern Med. 2009;169(2):10814. https://doi.org/10.1001/archinternmed.2008. 520 .

48. Salmon JW, Jiang R. E-prescribing: history, issues, and potentials. Online J Public Health Inform. 2012;4(3):ojphi.v4i3.4304. https://doi.org/10.5210/ ojphi.v4i3.4304.

49. Wang JK, Herzog NS, Kaushal R, Park C, Mochizuki C, Weingarten SR. Prevention of pediatric medication errors by hospital pharmacists and the potential benefit of computerized physician order entry. Pediatrics. 2007;119(1):e77-85. https://doi.org/10.1542/peds. 2006-0034.

50. Longhurst CA, Parast L, Sandborg CI, Widen E, Sullivan J, Hahn JS, et al. Decrease in hospital-wide mortality rate after implementation of a commercially sold computerized physician order entry system. Pediatrics. 2010;126(1):14-21. https://doi.org/10.1542/peds. 2009-3271.

51. Morriss FH, Abramowitz PW, Nelson SP, Milavetz G, Michael SL, Gordon SN, et al. Effectiveness of a Barcode Medication Administration System in reducing preventable adverse drug events in a neonatal intensive care unit: a prospective cohort study. J Pediatr. 2009;154:363-8.

52. Biltoft J, Finneman L. Clinical and financial effects of smart pump-electronic medical record interoperability at a hospital in a regional health system. Am J Health Syst Pharm. 2018;75(14):1064-8. https://doi.org/10. 2146/ajhp161058.

53. Smart pumps in practice: survey results reveal widespread use, but optimization is challenging. Institute for Safe Medication Practices. https://www.ismp.org/ resources/smart-pumps-practice-survey-results-revealwidespread-use-optimization-challenging. Accessed October 18, 2018.

54. Kankanhalli A, Hahn J, Tan S, Gao G. Big data and analytics in healthcare: introduction to the special section. Inf Syst Front. 2016;18(2):233-5. https://doi.org/ 10.1007/s10796-016-9641-2.

55.• Pollack MM, Holubkov R, Berg RA, Newth CJL, Meert $\mathrm{KL}$, Harrison RE, et al. Predicting cardiac arrests in pediatric intensive care units. Resuscitation. 2018;133:25-32. https://doi.org/10.1016/j. resuscitation.2018.09.019

This paper shows the potential of a multivariable model for predicting within 4 hours of admission to a PICU which patients are likely to suffer cardiac arrest, even days in advance.

56. Sutherland SM. Electronic health record-enabled bigdata approaches to Nephrotoxin-associated acute kidney injury risk prediction. Pharmacotherapy. 2018;38(8):804-12. https://doi.org/10.1002/phar. 2150 .
57. Rusin CG, Acosta SI, Shekerdemian LS, Vu EL, Bavare AC, Myers RB, et al. Prediction of imminent, severe deterioration of children with parallel circulations using real-time processing of physiologic data. J Thorac Cardiovasc Surg. 2016;152(1):171-7. https://doi.org/ 10.1016/j.jtcvs.2016.03.083.

58. Lloyd JK, Ahrens EA, Clark D, Dachenhaus T, Nuss KE. Automating a manual sepsis screening tool in a pediatric emergency department. Appl Clin Inform. 2018;9(4):803-8. https://doi.org/10.1055/s-00381675211 .

59. Cho I, Bates DW. Behavioral economics interventions in clinical decision support systems. Yearb Med Inform. 2018;27(1):114-21. https://doi.org/10.1055/s0038-1641221.

60. Reis AG, Nadkarni V, Perondi MB, Grisi S, Berg RA. A prospective investigation into the epidemiology of inhospital pediatric cardiopulmonary resuscitation using the international Utstein reporting style. Pediatrics. 2002;109(2):200-9.

61. Girotra S, Spertus JA, Li Y, Berg RA, Nadkarni VM, Chan PS, et al. Survival trends in pediatric in-hospital cardiac arrests: an analysis from get with the guidelines-resuscitation. Circ Cardiovasc Qual Outcomes. 2013;6(1):42-9. https://doi.org/10.1161/ CIRCOUTCOMES.112.967968.

62. Sharek PJ, Parast LM, Leong K, Coombs J, Earnest K, Sullivan J, et al. Effect of a rapid response team on hospital-wide mortality and code rates outside the ICU in a Children's hospital. JAMA. 2007;298(19):226774. https://doi.org/10.1001/jama.298.19.2267.

63. Chan PS, Jain R, Nallmothu BK, Berg RA, Sasson C. Rapid response teams: a systematic review and metaanalysis. Arch Intern Med. 2010;170(1):18-26. https:// doi.org/10.1001/archinternmed.2009.424.

64. Maharaj R, Raffaele I, Wendon J. Rapid response systems: a systematic review and meta-analysis. Crit Care. 2015;19:254. https://doi.org/10.1186/s13054-0150973-y.

65. Endsley MR. Toward a theory of situation awareness in dynamic systems. Hum Factors. 1995;37(1):32-64. https://doi.org/10.1518/001872095779049543.

66. Parshuram CS, Dryden-Palmer K, Farrell C, Gottesman R, Gray M, Hutchison JS, et al. Effect of a pediatric early warning System on all-cause mortality in hospitalized pediatric patients: the EPOCH randomized clinical trial. JAMA. 2018;319(10):1002-12. https://doi.org/10. 1001/jama.2018.0948.

67. Bonafide CP, Roberts KE, Priestley MA, Tibbetts KM, Huang E, Nadkarni VM, et al. Development of a pragmatic measure for evaluating and optimizing rapid response systems. Pediatrics. 2012;129(4):e874-81. https://doi.org/10.1542/peds.2011-2784.

68. Rothman MJ, Rothman SI, Beals J. Development and validation of a continuous measure of patient condition using the electronic medical record. J Biomed Inform. 2013;46(5):837-48. https://doi.org/10.1016/ j.jbi.2013.06.011. 
69. Brady PW, Muething S, Kotagal U, Ashby M, Gallagher $\mathrm{R}$, Hall $\mathrm{D}$, et al. Improving situation awareness to reduce unrecognized clinical deterioration and serious safety events. Pediatrics. 2013;131(1):e298-308. https://doi.org/10.1542/peds.2012-1364.

70. McClain Smith M, Chumpia M, Wargo L, Nicol J, Bugnitz M. Watcher initiative associated with decrease in failure to rescue events in pediatric population. Hosp Pediatr. 2017;7(12):710-5. https://doi.org/10. 1542/hpeds.2017-0042.

71. Gurses AP, Ozok AA, Pronovost PJ. Time to accelerate integration of human factors and ergonomics in patient safety. BMJ Qual Saf. 2012;21(4):347-51. https:// doi.org/10.1136/bmjqs-2011-000421.

72. System NlAoEaICoEatHC. In: Reid PP, Compton WD, Grossman JH, Fanjiang G, editors. Building a better delivery system: a new engineering/health care partnership. Washington (DC): National Academies Press (US); 2005.

73. Carayon P, Schoofs Hundt A, Karsh BT, Gurses AP, Alvarado CJ, Smith M, et al. Work system design for patient safety: the SEIPS model. Qual Saf Health Care. 2006;15(Suppl 1):i50-8. https://doi.org/10.1136/ qshc.2005.015842.

74. Holden RJ, Carayon P, Gurses AP, Hoonakker P, Hundt AS, Ozok AA, et al. SEIPS 2.0: a human factors framework for studying and improving the work of healthcare professionals and patients. Ergonomics. 2013;56(11):1669-86. https://doi.org/10.1080/ 00140139.2013 .838643$.

75. Schiff GD, Seoane-Vazquez E, Wright A. Incorporating indications into medication ordering-time to enter the age of reason. N Engl J Med. 2016;375(4):306-9. https://doi.org/10.1056/NEJMp1603964.

76. Falck S, Adimadhyam S, Meltzer DO, Walton SM, Galanter WL. A trial of indication based prescribing of antihypertensive medications during computerized order entry to improve problem list documentation. Int J Med Inform. 2013;82(10):996-1003. https://doi. org/10.1016/j.ijmedinf.2013.07.003.

77. Galanter W, Falck S, Burns M, Laragh M, Lambert BL. Indication-based prescribing prevents wrong-patient medication errors in computerized provider order entry (CPOE). J Am Med Inform Assoc. 2013;20(3):47781. https://doi.org/10.1136/amiajnl-2012-001555.

78. Walton SM, Galanter WL, Rosencranz H, Meltzer D, Stafford RS, Tiryaki F, et al. A trial of inpatient indication based prescribing during computerized order entry with medications commonly used off-label. Appl Clin Inform. 2011;2(1):94-103. https://doi.org/ 10.4338/ACI-2010-11-RA-0072.

79. Is an Indication-Based Prescribing System in Our Future? Institute for Safe Medication Practices. https:// www.ismp.org/resources/indication-based-prescribingsystem-our-future. Accessed October 22, 2018.

80. Hollnagel E, Wears R, Braithwaite J. From Safety I to Safety II: a white paper. 2015.

This white paper outlines the principles of Safety II and points a way forward toward improved patient safety.

81. Clay-Williams R, Hounsgaard J, Hollnagel E. Where the rubber meets the road: using FRAM to align work-asimagined with work-as-done when implementing clinical guidelines. Implement Sci. 2015;10:125. https://doi.org/10.1186/s13012-015-0317-y.

82. Grant S, Guthrie B. Efficiency and thoroughness tradeoffs in high-volume organisational routines: an ethnographic study of prescribing safety in primary care. BMJ Qual Saf. 2018;27(3):199-206. https://doi.org/ 10.1136/bmjqs-2017-006917.

83. Holden RJ, Rivera-Rodriguez AJ, Faye H, Scanlon MC, Karsh BT. Automation and adaptation: Nurses' problem-solving behavior following the implementation of bar coded medication administration technology. Cogn Tech Work. 2013;15(3):283-96. https://doi. org/10.1007/s10111-012-0229-4.

84. Fairbanks RJ, Wears RL, Woods DD, Hollnagel E, Plsek $\mathrm{P}$, Cook RI. Resilience and resilience engineering in health care. Jt Comm J Qual Patient Saf. 2014;40(8):376-83.

85. Merandi J, Vannatta K, Davis JT, McClead RE, Brilli R, Bartman T. Safety II behavior in a pediatric intensive care unit. Pediatrics. 2018;141(6):e20180018. https:// doi.org/10.1542/peds.2018-0018.

\section{Publisher's Note}

Springer Nature remains neutral with regard to jurisdictional claims in published maps and institutional affiliations. 